\title{
Nota técnica \\ EFECTO DEL CONSUMO DE DIETA LÍQUIDA Y ALIMENTO BALANCEADO SOBRE EL CRECIMIENTO Y DESARROLLO RUMINAL EN TERNERAS DE LECHERÍA ${ }^{1}$
}

\author{
Jorge Alberto Elizondo-Salazar ${ }^{2 / *}$, Melisa Sánchez-Álvarez** \\ Palabras clave: Rumen, leche, consumo, terneras, desarrollo ruminal. \\ Keywords: Rumen, milk, intake, calves, rumen development.

\section{RESUMEN}

La presente investigación tuvo como objetivo determinar el efecto del consumo de dieta líquida y alimento balanceado sobre el crecimiento y desarrollo ruminal en terneras de lechería. El experimento se llevó a cabo en una finca comercial ubicada a $2500 \mathrm{msnm}$ en la provincia de Cartago, Costa Rica. Diez animales de la raza Holstein se seleccionaron al nacimiento y se incluyeron aleatoriamente en 1 de 2 tratamientos. El tratamiento convencional consistió en ofrecer a los animales una dieta líquida de forma restringida (4 1.animal $\left.{ }^{-1} \cdot \mathrm{d}^{-1}\right)$. El tratamiento intensivo consistió en suministrar a los animales la dieta líquida en mayores cantidades, hasta alcanzar un consumo de 8 l.animal ${ }^{-1} \cdot \mathrm{d}^{-1}$. Se realizaron mediciones semanales de peso, altura a la cruz y a la cadera. Los animales se sacrificaron a las 8 semanas, con el fin de hacer evaluaciones y poder comparar el crecimiento y desarrollo ruminal. Los animales en el tratamiento intensivo presentaron menores consumos de alimento balanceado. No se encontraron diferencias significativas en cuanto al peso corporal, altura a la cruz o altura a la cadera entre tratamientos. Al brindar una mayor cantidad de leche con el

1 Proyecto 737-A9-184. Inscrito en la Vicerrectoría de Investigación. Universidad de Costa Rica.

2 Autor para correspondencia. Correo electrónico: jorge.elizondosalazar@ucr.ac.cr

\section{ABSTRACT}

Effect of liquid and solid diet intake on growth and rumen development of dairy calves. The objective of this study was to determine the effect of liquid and solid diet intake on growth and rumen development of dairy calves. The experiment was conducted in a commercial dairy farm located at 2500 m.a.s.l in the province of Cartago, Costa Rica. Ten Holstein calves were selected at birth and randomly included in 1 of 2 treatments. The conventional treatment consisted in providing calves a liquid diet on a restricted basis (4 1. calf $\left.^{-1} \cdot \mathrm{d}^{-1}\right)$. The intensive treatment consisted in supplying calves the same liquid diet in larger quantities, up to 8 l.calf ${ }^{-1} \cdot \mathrm{d}^{-1}$. Growth measurements including body weight, hip height and withers height were taken weekly. Calves were sacrificed at 8 weeks of age, in order to compare rumen growth and development. Animals in the intensive treatment had a significantly higher liquid diet intake and lower feed intake than calves in the conventional treatment. Growth measurements showed no significant differences between treatment groups. Calves in the intensive treatment had a poor rumen development and lesser papillae growth,

\footnotetext{
* Estación Experimental Alfredo Volio Mata. Facultad de Ciencias Agroalimentarias. Universidad de Costa Rica. Cartago, Costa Rica.

** Universidad Técnica Nacional, Sede Atenas. Alajuela, Costa Rica.
} 
tratamiento intensivo, se observó un menor desarrollo del rumen y del crecimiento de sus papilas, lo que puede afectar negativamente el desempeño posdesdete de los animales.

\section{INTRODUCCIÓN}

La crianza de reemplazos es un aspecto fundamental en cualquier sistema de producción lechero, ya que las terneras son las que van a sustituir en un determinado tiempo, a las vacas que poco a poco dejan la explotación. Reemplazos saludables, con tasas de crecimiento sostenidas para obtener tamaños adecuados y pesos meta, son 2 factores importantes que debe buscar cualquier sistema de crianza y desarrollo de terneras.

En Costa Rica, los sistemas intensivos de crianza y desarrollo de terneras de lechería, tradicionalmente se han basado en métodos que restringen la alimentación líquida diaria a una proporción de $10 \%$ del peso vivo del animal; así por ejemplo, si una ternera pesa $40 \mathrm{~kg}$, se le ofrece 4 litros de leche o reemplazador de leche al día. Este sistema restringido estimula al animal a consumir alimento balanceado durante los primeros días de vida con el fin de estimular el crecimiento y desarrollo funcional del rumen. Desde el punto de vista económico, ese sistema se ha recomendado para destetar a las terneras de forma temprana, con el fin de ahorrar dinero en mano de obra y en alimento líquido sin sacrificar la salud y el bienestar de las mismas. Sin embargo, durante los últimos años diversas investigaciones han mostrado mayores tasas de crecimiento y una mejor eficiencia alimenticia cuando se brindan cantidades mayores a las ofrecidas con el sistema convencional (Díaz et al. 2001, Jasper y Weary 2002, Khan et al. 2007a,b). Dicho sistema de alimentación se le ha denominado de distintas maneras: programa acelerado de crecimiento, programa de nutrición mejorada, programa de which may negatively affect calves' post-weaning performance.

nutrición intensificada o programa temprano de nutrición acelerada.

\section{Sistema de alimentación convencional versus acelerado}

Tal como se mencionó anteriormente, los sistemas de alimentación convencionales recomiendan ofrecer dieta líquida en una proporción de $10 \%$ del peso vivo de las terneras. Esta cantidad es mucho menor a aquella que los animales pudieran consumir si se les permitiera ingerirla a libre consumo. En otras palabras, cuando una ternera permanece con su madre o se le permite tomar leche de un sistema automático, típicamente mamará entre 6 y 10 veces al día, lo que le permitiría consumir leche en una proporción cercana al 20\% de su peso vivo (Albright y Arave 1997). Para ejemplificar la información anterior, considérese un experimento en el que a un grupo de terneras se les permitió permanecer con sus madres durante 2 semanas después del nacimiento para que mamaran libremente (Flower y Weary 2001); dentro del mismo experimento, a otro grupo control se les separó de sus madres en las primeras $24 \mathrm{~h}$ de vida y se les ofreció leche a razón de un $10 \%$ de su peso vivo por día durante 2 semanas, tal como se hace en el sistema de alimentación tradicional. Al final de las 2 semanas, las terneras que permanecieron con sus respectivas madres ganaron en promedio $16,5 \mathrm{~kg}$ en comparación con 4,5 kg que ganaron las terneras del grupo control. Básicamente, la gran diferencia se debe a que las terneras que tomaron leche de sus madres, lo hicieron en una proporción que osciló entre 16 y $24 \%$ de su peso corporal. 
En otro estudio llevado a cabo en Israel (Bar-Peled et al. 1997), se les ofreció a un grupo de terneras, reemplazador de leche en balde en cantidades convencionales, mientras que a otro grupo se les permitió mamar leche de sus madres durante 15 minutos 3 veces al día. Las terneras se destetaron a los 60 días y se observó que las terneras a las que se les permitió mamar, consumieron mayor cantidad de leche y obtuvieron pesos al destete y ganancias diarias de peso significativamente mayores que las terneras a las que se les ofreció reemplazador de leche en balde.

En un experimento llevado a cabo en Canadá (Jasper y Weary 2002), a un grupo de terneras se les alimentó leche integra de la forma convencional (10\% del peso corporal) y a otro grupo se les ofreció leche a libre consumo (ad libitum) con sistemas automáticos. Las terneras de ambos grupos se destetaron a las 6 semanas. En este estudio se pudo observar claramente que las terneras del segundo grupo consumieron más leche de la que normalmente se les permite tomar. En este caso, consumieron en promedio $8,79 \mathrm{~kg}$ de leche al día en comparación con 4,91 $\mathrm{kg}$ para el grupo control. Como resultado, las terneras que ingirieron más leche, consumieron en promedio menos alimento balanceado $90 \mathrm{~g} . \mathrm{d}^{-1} \mathrm{en}$ comparación con 170 g.d $\mathrm{d}^{-1}$. El efecto de un consumo mayor de leche resultó en mayores ganancias diarias de peso esto es, 780 g.d $\mathrm{d}^{-1}$ vs. 480 g.d $\mathrm{d}^{-1}, \mathrm{y}$ como resultado un peso al destete de $69,5 \mathrm{~kg}$ para las terneras que consumieron leche a libre consumo y $59,0 \mathrm{~kg}$ para las terneras que consumieron leche a razón de un $10 \%$ de su peso corporal. Con base en los resultados de estos y otros experimentos, lo que se refiere como crecimiento acelerado, es en realidad la restauración del crecimiento normal biológico en la vida temprana de las terneras, antes de que el consumo de concentrado sea la forma predominante de abastecimiento de nutrientes.

Es importante destacar que conforme se alimenta más cantidad de dieta líquida, el consumo de alimento balanceado decrece y un bajo consumo de éste se ha asociado con una disminución en la tasa de desarrollo y funcionalidad del rumen, lo que podría contribuir con el deterioro en la condición corporal de los animales cuando son destetados y alimentados con algún tipo de forraje (Jasper y Weary 2002, Davis et al. 2011).

A partir del análisis anterior, se identificó que en Costa Rica no existe algún estudio sobre este tipo de análisis, por lo que el objetivo de este experimento fue determinar el efecto del consumo de leche o reemplazador de leche y alimento balanceado sobre el crecimiento y desarrollo ruminal en terneras de lechería.

\section{MATERIALES Y MÉTODOS}

El experimento se llevó a cabo en una finca comercial ubicada a $2500 \mathrm{msnm}$ en la provincia de Cartago, con una precipitación anual promedio de $1750 \mathrm{~mm}$ y una temperatura promedio que ronda $\operatorname{los} 15,2^{\circ} \mathrm{C}$. Se seleccionaron al nacimiento, 8 terneras y 2 terneros de la raza Holstein que se incluyeron aleatoriamente en 1 de 2 tratamientos. El tratamiento 1 o convencional, consistió en ofrecer a los animales una dieta líquida de forma restringida (4 l.animal $\left.{ }^{-1} \cdot \mathrm{d}^{-1}\right)$, en 2 tomas diarias (2 1 a.m y 21 p.m), a partir del segundo día de edad. El tratamiento 2 o intensivo, consistió en suministrar a los animales la dieta líquida, en mayores cantidades, hasta alcanzar un consumo de $81 . \mathrm{d}^{-1}$ (4 1 a.m y 41 p.m).

Todos los animales al nacimiento fueron separados de la madre y pesados. Se les curó el ombligo con tintura de yodo al 7\%. A las vacas se les ordeñó el calostro y se determinó la calidad del mismo, con ayuda de un calostrómetro (Biogenics, Oregon). Cuando la concentración de inmunoglobulinas del calostro de la madre fue inferior a 50 g..$^{-1}$, se utilizó calostro de un banco de calostro. A cada animal se le ofreció 4 litros de calostro con chupón en las primeras 2 horas de vida. Aquellos individuos que no consumieron los 4 litros de calostro por medio del chupón, se les suministró el mismo con un alimentador esofágico (Elizondo et al. 2011). Todos los animales fueron alojados en jaulas individuales. Durante el primer mes de vida, se les ofreció leche íntegra (12,5\% MS; 3,1\% PC.kg-1 MS; 5,37 Mcal de 
EM. $\mathrm{kg}^{-1} \mathrm{MS}$ ), por medio de un balde, de acuerdo con las cantidades establecidas para cada tratamiento. Luego, se les suministró un reemplazador de leche comercial (97\% MS; 20,5\% PC. $\mathrm{kg}^{-1} \mathrm{MS}$; $16 \%$ grasa. $\mathrm{kg}^{-1} \mathrm{MS} ; 43 \%$ lactosa.kg-1 MS; 4,04 Mcal de EM.kg-1 MS) reconstituido a 0,125 kg. $\mathrm{l}^{-1}$. Los animales se destetaron a las 8 semanas, sin embargo a las 7 semanas se redujo la dieta líquida a la mitad, en una sola toma por la mañana, con el fin de llevar a cabo un destete menos traumático. Todos los animales recibieron alimento balanceado (Cuadro 1) tipo iniciador a partir del segundo día, con el aporte de pequeñas cantidades, estimulándoles el consumo durante los primeros días. Se llevaron registros tanto del alimento ofrecido como del rechazado de manera semanal. Igualmente, se llevó un control detallado de los precios de los alimentos ofrecidos con el fin de establecer el valor económico de cada dieta.

Para llevar registros del crecimiento de los animales, se realizaron mediciones semanales de peso, altura a la cruz y a la cadera, siempre el mismo día de la semana y a la misma hora, para evitar inconsistencias y reducir el error (Khan et al. 2007a). Cada tratamiento incluyó un macho, que se sacrificó al destete (8 semanas), con el fin de hacer evaluaciones y poder comparar el crecimiento y desarrollo ruminal, entre los 2 tratamientos. El procedimiento utilizado para medir el desarrollo ruminal fue el establecido por Lesmeister et al. (2004), donde el retículo-rumen se abrió en un plano casi simétrico a la derecha e izquierda separadas por la porción del rumen que se mantuvo intacta. Posteriormente para el muestreo de las papilas, el rumen se separó en 9 zonas de muestreo diferentes, a saber: porción caudal del saco ciego caudo-ventral, lado derecho de saco caudo-dorsal, lado izquierdo del saco caudo-dorsal, el lado derecho del saco crano-dorsal, lado izquierdo del saco craneodorsal, lado derecho del saco cráneo-ventral, lado izquierdo del saco craneo-ventral, lado derecho de la porción ventral del saco ciego caudo-ventral y parte izquierda de la porción ventral del saco ciego caudo-ventral.

Se tomó una muestra de aproximadamente $1,0 \mathrm{~cm}^{2}$ de tejido del centro de cada área y 4 muestras más de las esquinas de cada zona esto es, 5 muestras por zona. Las muestras fueron fijadas en solución de formaldehído al 30\% para las mediciones posteriores.

De cada muestra de tejido por zona, se seleccionaron al azar, 5 papilas (25 por zona), a las cuáles se les realizaron mediciones de altura $\mathrm{y}$ ancho de las papilas, y espesor de la pared del rumen. Las mediciones de las papilas y de la pared del rumen fueron tomadas con la ayuda de un estereoscopio (Zeiss, modelo Sterri) con aumento de 2000-c y un vernier digital de precisión de $0,01 \mathrm{~mm}$.

Los datos de consumos, pesos y medidas de los animales para los diferentes tratamientos fueron analizados, por medio del análisis de medidas repetidas y el procedimiento MIXED de

Cuadro 1. Perfil nutricional de alimento balanceado.

\begin{tabular}{llr}
\hline \multicolumn{1}{c}{ Nutriente } & Nivel & Valor \\
\hline Humedad, \% & Máximo & 13,00 \\
Proteína cruda, $\%$ & Mínimo & 18,00 \\
Extracto etéreo, \% & Mínimo & 3,50 \\
Fibra cruda, $\%$ & Máximo & 7,00 \\
Energía digestible, Mcal. $\mathrm{kg}^{-1}$ & Mínimo & 3,30 \\
Energía metabolizable Mcal. $\mathrm{kg}^{-1}$ & Mínimo & 2,19 \\
Calcio; $\%$ & Mínimo & 0,75 \\
Calcio, $\%$ & Máximo & 1,00 \\
Fósforo, $\%$ & Mínimo & 0,45 \\
Sal $(\mathrm{NaCl}), \%$ & Mínimo & 0,50 \\
Sal $(\mathrm{NaCl}), \%$ & Máximo & 0,80 \\
\hline
\end{tabular}

Agronomía Costarricense 36(2): 81-90. ISSN:0377-9424 / 2012 
SAS (2006) donde el animal se tomó como variable aleatoria, y se efectuó la separación de medias con la prueba de Waller-Duncan en aquellas variables que resultaron significativas. Los datos del rumen fueron analizados a través del procedimiento GLM de SAS (2006), sin embargo, no se efectuó separación de medias ya que solamente se utilizaron los datos de 2 animales.

\section{RESULTADOS Y DISCUSIÓN}

\section{Consumo de dieta líquida y alimento}

La información referente al consumo de alimento registrado durante el periodo experimental se presenta de forma resumida en el Cuadro 2. Según los resultados, los animales que tomaron mayor cantidad de dieta líquida, presentaron menores consumos de alimento balanceado $(\mathrm{p}<0,05)$, especialmente durante las últimas 2 semanas; resultados similares se obtuvieron en un experimento en Canadá (Jasper y Weary 2002), donde un grupo de terneras se alimentaron con leche íntegra (10\% del peso corporal) y a otro grupo se les ofreció leche a libre consumo en sistemas automáticos. En dicho estudio, las terneras del segundo grupo consumieron en promedio 8,79 1 de leche al día y tuvieron consumos menores de alimento. Esta situación es típica, ya que se ha demostrado que animales que consumen mayores cantidades de dieta líquida presentan un llenado físico, por lo que no experimentan la necesidad de consumir cantidades mayores de alimento balanceado (Jasper y Weary 2002).

En el Cuadro 2 se aprecia también los costos de cada dieta. Puede observarse claramente que la dieta líquida representó un costo mayor para el tratamiento intensivo y el alimento balanceado un costo mayor para el tratamiento convencional. Sin embargo, al considerar el costo total, el tratamiento convencional resultó 928 colones (US\$1,85) más barato que el intensivo dicho de otra forma 21.544 vs. 22.472 colones, respectivamente. Davis et al. (2011), reportan US\$1,27 más para un programa de alimentación intensivo en comparación con uno tradicional.

Lesmeister y Henrichs (2005) reportan un consumo semanal de $3563 \mathrm{~g}$ de un alimento texturizado con $5 \%$ de melaza a las 6 semanas. Por su parte, Suárez et al. (2006) reportan consumos semanales que van desde los 2800 hasta los $4900 \mathrm{~g}$ de alimento balanceado a las 8 semanas

Cuadro 2. Consumo de dieta líquida y alimento balanceado (MS) semanal en terneras alimentadas con las diferentes dietas experimentales.

\begin{tabular}{|c|c|c|c|c|}
\hline \multirow[b]{2}{*}{ Semana } & \multicolumn{2}{|c|}{ Convencional } & \multicolumn{2}{|c|}{ Intensivo } \\
\hline & Leche, 1 & Alimento, $\mathrm{g}$ & Leche, 1 & Alimento, $\mathrm{g}$ \\
\hline 1 & $4,0^{\mathrm{a}}$ & 147 & $5,0^{\mathrm{b}}$ & 127 \\
\hline 2 & $4,0^{\mathrm{a}}$ & 347 & $6,0^{\mathrm{b}}$ & 373 \\
\hline 3 & $4,0^{\mathrm{a}}$ & 1225 & $7,0^{\mathrm{b}}$ & 1299 \\
\hline 4 & $4,0^{\mathrm{a}}$ & 1415 & $8,0^{\mathrm{b}}$ & 1565 \\
\hline 5 & $4,0^{\mathrm{a}}$ & 2359 & $8,0^{\mathrm{b}}$ & 2088 \\
\hline 6 & $4,0^{\mathrm{a}}$ & 3499 & $8,0^{\mathrm{b}}$ & 2397 \\
\hline 7 & $4,0^{\mathrm{a}}$ & $4924^{\mathrm{a}}$ & $8,0^{\mathrm{b}}$ & $2559^{b}$ \\
\hline 8 & $4,0^{\mathrm{a}}$ & $6701^{a}$ & $4,0^{\mathrm{a}}$ & $4137^{b}$ \\
\hline EEM & 0,0 & 898 & 0,0 & 733 \\
\hline Costo, colones & $7267^{\mathrm{a}}$ & $14277^{b}$ & $12400^{\mathrm{b}}$ & $10072^{\mathrm{a}}$ \\
\hline
\end{tabular}

\footnotetext{
${ }^{\text {ab }}$ Valores en una misma hilera con diferente letra son diferentes, $\mathrm{p}<0,05$.

EEM=Error estándar de la media. Tasa de cambio 1 US $\$=500$ colones.
} 
en terneros que se suplementaron con diferentes tipos de alimentos balanceados.

Cuando se consideró el consumo de nutrientes de las 2 dietas, se encontraron diferencias significativas $(\mathrm{p}<0,05)$, como lo muestra el Cuadro 3. Con el tratamiento convencional los animales consumieron la mayor cantidad de nutrientes durante las últimas 2 semanas del experimento. En otros ensayos se han obtenido mayores consumos de nutrientes con el tratamiento intensivo, pero esto se ha debido a que el reemplazador de leche utilizado ha sido nutricionalmente superior al suministrado en el presente experimento (Jasper y Weary 2002).

\section{Peso y crecimiento de los animales}

Los pesos de los animales registrados durante el estudio, no mostraron diferencias significativas entre tratamientos (Cuadro 4). En otros estudios, se determinó que incrementar el consumo de energía y proteína en terneras con sistemas intensivos de alimentación líquida, mejoró la ganancia de peso y el desarrollo esquelético en los animales. Así por ejemplo, Jasper y Weary (2002) reportaron una diferencia de 10,5

Cuadro 3. Consumo de proteína cruda y energía metabolizable semanal en terneras alimentadas con las diferentes dietas experimentales.

\begin{tabular}{ccccc}
\hline & \multicolumn{2}{c}{ Convencional } & \multicolumn{2}{c}{ Intensivo } \\
Semana & PC, $g$ & EM, Mcal & PC, & EM, Mcal \\
\hline 1 & 42 & 3,00 & 42 & 4,63 \\
2 & 78 & 3,44 & 90 & 7,84 \\
3 & 236 & 5,37 & 261 & 7,47 \\
4 & 357 & 5,12 & 487 & 8,61 \\
5 & 527 & 7,19 & 581 & 9,28 \\
6 & 732 & 9,68 & 636 & $9,64^{\mathrm{b}}$ \\
7 & $989^{\mathrm{a}}$ & $12,80^{\mathrm{a}}$ & $666^{\mathrm{b}}$ & $11,08^{\mathrm{b}}$ \\
\hline
\end{tabular}

${ }^{a b}$ Valores en una misma hilera con diferente letra son diferentes, $\mathrm{p}<0,05$.

EEM=Error estándar de la media.

Cuadro 4. Peso de las terneras $(\mathrm{kg} \pm \mathrm{DE})$ alimentadas con las diferentes dietas.

\begin{tabular}{|c|c|c|}
\hline \multirow[b]{2}{*}{ Semana } & \multicolumn{2}{|c|}{ Tratamiento } \\
\hline & Convencional & Intensivo \\
\hline Nacimiento & $42 \pm 4$ & $41 \pm 3$ \\
\hline 1 & $48 \pm 3$ & $43 \pm 4$ \\
\hline 2 & $50 \pm 4$ & $46 \pm 4$ \\
\hline 3 & $51 \pm 3$ & $49 \pm 3$ \\
\hline 4 & $52 \pm 3$ & $53 \pm 4$ \\
\hline 5 & $54 \pm 4$ & $59 \pm 3$ \\
\hline 6 & $55 \pm 4$ & $63 \pm 4$ \\
\hline 7 & $57 \pm 4$ & $65 \pm 4$ \\
\hline 8 & $62 \pm 5$ & $68 \pm 4$ \\
\hline
\end{tabular}


$\mathrm{kg}$ al destete a favor de animales que consumieron mayor cantidad de leche. Por su parte, RaethKnight et al. (2009) encontraron diferencias de alrededor de $6 \mathrm{~kg}$ a los 42 días a favor de animales que se alimentaron con cantidades mayores de reemplazador de leche.

Davis et al. (2011) llevaron a cabo un estudio en terneras con un programa de alimentación intensivo versus uno convencional con el fin de determinar su efecto sobre las tasas de crecimiento en la etapa pre- y posdestete. Los autores reportaron diferencias de $9 \mathrm{~kg}$ a los 42 días y $6 \mathrm{~kg}$ a los 56 días a favor de los animales en el programa de alimentación intensivo, pero encontraron que dicha diferencia se había perdido a las 12 semanas, lo que sugiere que las terneras en el programa intensivo tuvieron un menor desarrollo ruminal, con menores tasas de crecimiento en la etapa posdestete, cuando se les ofreció dietas altas en forraje.
En el presente ensayo, los animales en el tratamiento intensivo no superaron el peso de los animales en el tratamiento convencional, aunque los animales del tratamiento convencional presentaron mayor consumo de nutrientes a partir de la sétima semana.

Vale señalar que los pesos obtenidos en el presente ensayo son muy similares a los obtenidos en otras latitudes. Coverdale et al. 2004 reportan pesos entre 58,7 y $65,6 \mathrm{~kg}$ a los 52 días. Khan et al. (2007a) y encontraron pesos entre los 64,13 y $68,71 \mathrm{~kg}$ en animales destetados a las 7 semanas, mientras que Suárez et al. (2011) reportan pesos entre 45,9 y $47,1 \mathrm{~kg}$ a las 5 semanas.

Cuando se considera la altura a la cruz y a la cadera, no se encontraron diferencias significativas $(p>0,05)$ entre tratamientos (Cuadro 5). Situación similar reportaron Raeth-Knight et al. (2009), donde la altura a la cadera fue alrededor de $91,2 \mathrm{~cm}$ a los 56 días.

Cuadro 5. Altura a la cruz $(\mathrm{cm})$ y a la cadera $(\mathrm{cm})$ de las terneras alimentadas con los diferentes tratamientos.

\begin{tabular}{ccccc}
\hline & \multicolumn{2}{c}{ Convencional } & Tratamiento & \multicolumn{2}{c}{ Intensivo } \\
Semana & Altura a la cruz & Altura a la cadera & Altura a la cruz & Altura a la cadera \\
\hline Nacimiento & 77,0 & 81,2 & 76,0 & 80,3 \\
1 & 79,8 & 83,8 & 76,2 & 81,5 \\
2 & 82,2 & 86,0 & 80,0 & 86,2 \\
3 & 83,8 & 87,0 & 81,5 & 87,5 \\
4 & 85,0 & 89,0 & 86,0 & 88,8 \\
5 & 86,5 & 90,5 & 87,2 & 90,3 \\
6 & 87,8 & 91,5 & 88,3 & 92,5 \\
7 & 88,5 & 92,0 & 89,5 & 93,7 \\
EEM & 90,0 & 93,2 & 91,2 & 94,8 \\
\hline
\end{tabular}

EEM:Error estándar de la media.

\section{Desarrollo ruminal}

Los datos obtenidos para la evaluación del desarrollo ruminal, se pueden observar en los Cuadros 6 y 7. Puede apreciarse claramente cómo los animales que consumieron menor cantidad de dieta líquida y más alimento balanceado, presentaron el mayor desarrollo ruminal, lo que concuerda con muchas de las especulaciones respecto a los programas de crecimiento acelerado.

La altura y el ancho de las papilas ruminales fue mayor en el tratamiento convencional que en el tratamiento intensivo; igualmente, el grosor de la pared ruminal fue mayor en los animales que consumieron menor cantidad de leche. Estos resultados concuerdan con observaciones 
Cuadro 6. Peso (g) de los compartimentos estomacales en los animales para los diferentes tratamientos.

\begin{tabular}{lcc}
\hline & & Tratamiento \\
Compartimento & Convencional & Intensivo \\
\hline Retículo-Rumen & 1345 & 647 \\
Omaso & 302 & 156 \\
Abomaso & 427 & 431 \\
\hline
\end{tabular}

Cuadro 7. Efecto del tratamiento sobre la altura y ancho de las papilas, y el grosor de la pared ruminal.

\begin{tabular}{cccc}
\hline Tratamiento & Altura $(\mathrm{mm})$ & Ancho $(\mathrm{mm})$ & Grosor $(\mathrm{mm})$ \\
\hline Convencional & $2,68 \pm 0,01$ & $1,19 \pm 0,10$ & $2,58 \pm 0,53$ \\
Intensivo & $0,78 \pm 0,02$ & $0,47 \pm 0,02$ & $2,28 \pm 0,57$ \\
\hline
\end{tabular}

encontradas por otros autores; por ejemplo un estudio para determinar el efecto de la edad del destete sobre el desarrollo ruminal en terneras de lechería, Kehoe et al. (2007), destetaron animales a las 3, 4, 5 ó 6 semanas y los sacrificaron a las 8 semanas para evaluar la altura y el ancho de las papilas, y el grosor de la pared ruminal. Los autores no encontraron diferencias estadísticas significativas entre edades de destete y el promedio de las medidas obtenidas fue de 2,44; 1,08 y 2,09 mm, respectivamente. Dichos valores se encuentran por debajo de los obtenidos en el presente estudio con el tratamiento convencional pero son superiores a los obtenidos para el tratamiento intensivo.

En otro experimento, Suárez et al. (2011) estudiaron el efecto de 2 niveles de reemplazador de leche $\left(0,64\right.$ vs. 0,94 kg. $\left.\mathrm{d}^{-1}\right)$ sobre el desarrollo ruminal en terneras. Los autores sacrificaron los animales a las 5 semanas y encontraron diferencias significativas entre tratamientos para la altura de las papilas (1,10 vs. $0,78 \mathrm{~mm}$ ) y ancho de las papilas $(0,45$ vs. $0,38 \mathrm{~mm})$. Para el grosor de la pared ruminal no encontraron diferencias significativas (1,57 vs. $1,64 \mathrm{~mm}$ ). Los investigadores concluyeron que el mayor desarrollo ruminal fue para los animales que consumieron menor cantidad de reemplazador y mayor cantidad de alimento balanceado, tal como se determinó en el presente ensayo.

En el pasado, diversos estudios se abocaron a valorar el efecto de la inclusión de alimentos balanceados en las dietas para terneras, con el fin de evaluar el desarrollo ruminal, ya que se pensaba que el consumo de forraje era esencial para el desarrollo del retículo-rumen. Luego, se descubrió que el alimento seco, especialmente aquel con un alto potencial de fermentación, producía un mayor estímulo, para el desarrollo más rápido de los tejidos de los pre-estómagos ya que los alimentos balanceados son digeridos y convertidos a ácido propiónico, butírico y acético, que son ácidos grasos volátiles que resultan de la fermentación microbial de los carbohidratos y proteínas en el rumen. El estímulo principal para el desarrollo de las papilas es particularmente el ácido butírico y el ácido propiónico (Flatt et al. 1958, Tamate et al. 1962). Por su parte, las terneras alimentadas únicamente con dietas líquidas, exhiben un desarrollo anormal de los preestómagos (Khan et al. 2007a,b). A pesar de que puede observarse algún desarrollo, las paredes son delgadas y el desarrollo papilar retardado (Tamate et al. 1962). Debe además hacerse hincapié en el poco efecto que ejerce la leche o reemplazador de leche a nivel ruminal, ya que la gotera esofágica dirige dichos 
líquidos hacia el abomaso, que evita cualquier estímulo a nivel ruminal. Por esta razón, el rumen de terneras alimentadas solamente con leche o reemplazador de leche permanecerá pequeño y el crecimiento y desarrollo de las papilas y paredes del rumen se verán restringidos, a pesar de que se alimenten con cantidades crecientes de estos alimentos líquidos. Sin embargo, cuando los animales comienzan a consumir alimento iniciador a una edad temprana, los pre-estómagos aumentan rápidamente en volumen, peso, músculo y capacidad de absorción (Anderson et al. 1987).

Para lograr un adecuado desarrollo del rumen es recomendable suministrar calostro de buena calidad a las terneras durante las primeras horas de vida, ya sea con chupón o con alimentador esofágico, pues algunas de las terneras a las que se les permite mamar directamente de la vaca, no consumen suficiente calostro o el mismo no es de buena calidad; se recomienda alimentar con cantidades moderadas de leche o reemplazador, a razón de $10 \%$ del peso vivo al nacimiento y continuar con esta recomendación hasta una semana antes del destete. En ese momento se recomienda alimentar a razón de un $5 \%$ en una sola toma. Independientemente de que las terneras consuman leche o reemplazador de leche; además se debe suministrar agua fresca a libre consumo, a partir del tercer día y alimento balanceado (concentrado iniciador), en pequeñas cantidades, a partir del tercer día y finalmente recordar que el destete de las terneras debe basarse en el adecuado desarrollo del rumen y no en la edad del animal.

\section{CONCLUSIONES Y RECOMENDACIONES}

Los animales consumieron menor cantidad de alimento balanceado al consumir mayor cantidad de leche bajo el sistema intensivo y según lo anterior, se observó un menor desarrollo del rumen así como del crecimiento de las papilas ruminales. Por otra parte, no se encontraron diferencias significativas entre los tratamientos, al considerar la ganancia de peso, la altura a la cadera y la altura a la cruz de los animales. Bajo las condiciones en que se llevó a cabo el experimento, los animales que consumieron menor cantidad de leche y mayor cantidad de alimento balanceado presentaron las mejores características a nivel ruminal, por lo que se espera que bajo este sistema, los animales puedan destetarse a una edad de 8 semanas, sin que repercuta negativamente en el periodo pos-destete, ya que están anatómica y fisiológicamente mejor adaptados para consumir dietas a base de forraje y concentrado.

\section{LITERATURA CITADA}

ALBRIGHT L., ARAVE C. 1997. The behaviour of cattle. CAB International, Wallingford, UK. 366 p.

ANDERSON K., NAGARAJA T., MORRIL J. 1987. Ruminal and metabolic development in calves weaned conventionally or early. J. Dairy Sci. 70:1000-1005.

BAR-PELED U., ROBINZON B., MALTZ E., TAGARI H., FOLMAN Y., BRUCKENTAL L., VOET H., GACITUA H., LEHRER R. 1997. Increased weight gain and effects on production parameters of Holstein heifer calves that were allowed to suckle from birth to six weeks of age. J. Dairy Sci. 80:2523-2528.

COVERDALE J., TYLER H., QUIGLEY J., BRUMM J. 2004. Effect of various levels of forage and form of diet on rumen development and growth in calves. J. Dairy Sci. 87:2554-2562.

DAVIS L., VANDEHAAR M., WOLF C., LIESMAN J., CHAPIN L., WEBER M. 2011. Effect of intensified feeding of heifer calves on growth, pubertal age, calving age, milk yield, and economics. J. Dairy Sci. 94:3554-3567.

DIAZ M., VAN AMBURG M., SMITH J., KELSEY J., HUTTEN E. 2001. Composition of growth of Holstein calves fed milk replacer from birth to 105-kilogram body weight. J. Dairy sci. 84:830-842.

ELIZONDO J., JONES C., HEINRICHS A. 2011. Feeding colostrum with an esophageal feeder does not reduce immunoglobulin $\mathrm{G}$ absorption in neonatal dairy heifer calves. Professional Animal Scientist. 27:561564.

FLATT W., WARNER R., LOOSLI J. 1958. Influence of purified materials on the development of the ruminant stomach. J. Dairy Sci. 41:1593-1600.

FLOWER F., WEARY D. 2001. Effects of early separation on the dairy cow and calf: 2. Separation at 1 day and 2 weeks after birth. Appl. Anim. Behav. Sci. 70:275-284

JASPER J., WEARY D. 2002. Effects of ad libitum milk intake on dairy calves. J. Dairy Sci. 85:3054-3058.

KEHOE S., DECHOW C., HEINRICHS J. 2007. Effects of weaning age and milk feeding frequency on dairy 
calf growth, health and rumen parameters. Livestock Science. 110:267-272.

KHAN M., LEE H., LEE W., KIM H., KIM K., HUR T., SUH G., KANG S., CHOI Y. 2007a. Structural growth, rumen development, and metabolic and immune responses of Holstein male calves fed milk through step down and conventional methods. J. Dairy Sci. 90:3376-3387.

KHAN M., LEE H., LEE W., KIM H., KIM K., KI J., HA H., LEE G., CHOI Y. 2007b. Pre- and post-weaning performance of Holstein female calves fed milk through step-downs and conventional methods. J. Dairy Sci. 90:876-885.

LESMEISTER K., HEINRICHS A. 2005. Effects of adding extra molasses to a texturized calf starter on rumen development, growth characteristics, and blood parameters in neonatal dairy calves. J. Dairy Sci. 88:411-418.

LESMEISTER K., TOZER P., HEINRICHS A. 2004. Development and analysis of a rumen. Tissue sampling procedure. J. Dairy Sci. 87:1336-1344.

RAETH-KNIGHT M., CHESTER-JONES H., HAYES S., LINN J., LARSON R., ZIEGLER D., ZIEGLER
B. 2009. Impact of conventional or intensive milk replacer programs on Holstein heifer performance through six months of age and during first lactation. J. Dairy Sci. 92:799-809.

SAS INSTITUTE. 2006. SAS/STAT 9.1 User`s Guide. Version 9.1 ed. SAS Institute Inc. Cary. N.C. U.S.A. $5121 \mathrm{p}$.

SUÁREZ B., VAN REENEN C., GERRITS W., STOCKHOFE N., VAN VUUREN A., DIJKSTRA

J. 2006. Effects of supplementing concentrates differing in carbohydrate composition in veal calf diets: II. Rumen development. J. Dairy Sci. 89:43764386.

SUÁREZ F., HILL T., HEINRICHS A., BATEMAN H., ALDRICH J., SCHLOTTERBECK R. 2011. Effects of including corn distillers dried grains with solubles in dairy calf feeds. J. Dairy Sci. 94:3037-3044.

TAMATE H., McGILLIARD A., JACOBSON N., GETTY R. 1962. Effect of various dietaries on the anatomical development of the stomach in the calf. J. Dairy Sci. 45:408-420. 